9. J. Milnor and E. Spanier, Two remarks on fiber homotopy type, Pacific J. Math. 10 (1960), 505-509. MR 22 \#8524.

10. D. Sullivan and C. P. Rourke, On the Kervaire obstruction, Ann. of Math. (2) 94 (1971), 397-413. MR 46 \#4546.

11. C. T. C. Wall, Surgery on compact manifolds, Academic Press, New York, 1970.

DEPARTMENT OF MATHEMATICS, UNIVERSITY OF MARYLAND, COLLEGE PARK, MARYLAND 20742

PROCEEDINGS OF THE

AMERICAN MATHEMATICAL SOCIETY

Volume 50, July 1975

\title{
THE INTERSECTION MULTIPLICITY OF $n$-DIMENSIONAL PARACOMPACT SPACES
}

\author{
GLENN P. WELLER
}

ABSTRACT. It is shown that there is an integer $\nu(n) \leq 3^{2 n+1}-1$ such that any $n$-dimensional paracompact space $X$ has intersection mulo tiplicity at most $\nu(n)$. That is, if $\mathcal{U}$ is an open cover of $X$, then there is an open cover $\theta$ refining $U$ such that any element of $\mathcal{V}$ intersects at most $\nu(n)$ elements of $\mathcal{O}$.

For any open cover $\mathcal{U}$ of a topological space $X$ define $m(\mathcal{U})$ to be the maximum number of elements of $\mathcal{U}$ that any member of $\mathcal{U}$ can intersect. $X$ is said to have intersection multiplicity at most $m$ if every open cover $\mathcal{U}$ of $X$ has an open refinement $(\mathcal{O}$ covering $X$ such that $m(\mathcal{O}) \leq m$. The intersection multiplicity of $X$ is then the least integer $m$ such that $X$ has intersection multiplicity at most $m$ and is denoted $m(X)$. Intersection multiplicity is clearly preserved by homeomorphisins. Also if $A$ is a closed subset of $X$, then $m(A) \leq m(X)$ 。

Received by the editors February 15, 1974 and, in revised form, May 7, 1974. $55 \mathrm{C} 10$.

AMS (MOS) subject classifications (1970). Primary 57C05, 54D20, 54F 45,

Key words and phrases. Intersection multiplicity, paracompact spaces, simplicial complex, dimension. 
Theorem. For every positive integer $n$ there is a positive integer $\nu(n)$ $\leq 3^{2 n+1}-1$ such that, if $X$ is an $n$-dimensional paracompact space, then $m(X) \leq \nu(n)$.

This Theorem generalizes the result proved in [3] for compact $n$-dimensional metric spaces. The Theorem is a consequence of the following lemmas.

Lemma 1. If $R^{P}$ is $p$-dimensional Euclidean space, then $m\left(R^{P}\right) \leq$ $3^{P}-1$.

Lemma 2. If $K$ is an $n$-dimensional simplicial complex, then $m(K) \leq$ $3^{2 n+1}-1$.

Proof of the Theorem. Suppose $\mathcal{U}$ is an arbitrary open cover of $X$. Then $\mathcal{U}$ has a refinement $\mathcal{O}$ of order $\leq n$ and the nerve $N(\mathcal{O})$ of $\mathcal{O}$ is a simplicial complex of dimension $\leq n$. Since $X$ is paracompact there is a canonical (or nerve) map $\mathcal{F}: X \rightarrow N(\mathcal{O})$. See [2, p. 152]. If $\mathcal{S}$ denotes the cover of $N(\mathcal{O})$ by open stars of vertices, then $\mathcal{F}^{-1}(\mathfrak{S})$ is a cover of $X$ refining $\mathcal{O}$ and hence $\mathcal{U}$.

By Lemma $2, m(N(\mathcal{O})) \leq 3^{2 n+1}-1$. Hence $\mathcal{S}$ has a refinement $t^{n}$ with $m(\mathfrak{W}) \leq 3^{2 n+1}-1$. So $m(\mathcal{F}-1(7 \mathfrak{i})) \leq 3^{2 n+1}-1$ and $\mathcal{F}^{-1}(\mathcal{C})$ refines $\mathfrak{F}^{-1}(\mathfrak{S})$ and hence $U$. The proof is thus complete.

Proof of Lemma 2. $K$ can be embedded as a closed subset of $R^{2 n+1}$ by $\left[1\right.$, p. 215]. So by Lemma $1, m(K) \leq m\left(R^{2 n+1}\right) \leq 3^{2 n+1}-1$.

Proof of Lemma 1. Let $\mathcal{O}$ be the cover of $R^{\bar{P}}$ consisting of open cubes of side length 2 and centered at integer lattice points. Two such cubes can intersect only when at least one pair of the coordinates of their centers differs by at most the integer one. Hence, $m\left(\mathcal{O}^{0}\right)=3^{P}-1$.

Let $\mathcal{U}$ be an arbitrary open cover of $R^{P}$. The proof is completed by exhibiting a squeezing homeomorphism $b: R^{P} \rightarrow R^{P}$ such that $b(\mathcal{O})$ refines U.

Let $R^{P}$ have the norm given by $\|x\|=\max \left\{\mid x_{i} \| 1 \leq i \leq p\right\}$ where $x=$ $\left(x_{1}, \cdots, x_{p}\right)$. Let $C(i)$ denote the cube $\{x \mid\|x\| \leq i\}$. There is no loss of generality in assuming that if $U \in \mathcal{U}$ and $U \cap C(i) \neq \varnothing$ then $U$ is contained in the interior of $C(i+1)$.

Let $\delta_{i}$ be a Lebesgue number of the cover $\mathcal{U}$ restricted to $C(i+2)$ with respect to the above norm. We will construct the homeomorphism $b$ such that if $V \in \mathcal{O}$ and $h(V) \cap C(i)=\varnothing$ then the diameter of $b(V)<\delta_{i}$. If the sequence $\left\{\delta_{i}\right\}$ is chosen to be decreasing, then $b(V) \subset U$ for some $U \in \mathcal{U}$. 
One such homeomorphism $b$ can be defined by.

$$
b\left(x_{1}, \cdots, x_{p}\right)=\left(\mathcal{F}\left(x_{1}\right), \cdots, \mathcal{F}\left(x_{p}\right)\right)
$$

where $\mathcal{F}: R^{1} \rightarrow R^{1}$ is an increasing homeomorphism having the property: for each $i>0$ and each integer $n$, if $\mathcal{F}[n, n+2] \cap[-i, i]=\varnothing$, then the length of $\mathcal{F}[n, n+2]<\delta_{i}$.

One particular function $\mathcal{F}$ can be constructed as follows: Let $m_{0}=0$ and inductively find integers $m_{i}$ such that $m_{i+2}-m_{i+1} \geq m_{i+1}-m_{i} \geq 2$ and $\left(m_{i+1}-m_{i}\right)^{-1}<1 / 2 \delta_{i}$. Then for $x \geq 0$ satisfying $m_{i} \leq x \leq m_{i+1}$ define $\mathfrak{F}(x)=i+\left(x-m_{i}\right)\left(m_{i+1}-m_{i}\right)^{-1}$. For $x \leq 0$ define $\mathcal{F}(x)=-\mathfrak{F}(-x)$. It can be checked that $\mathcal{F}$ has the property mentioned above.

Acknowledgment. The author wishes to thank the referee for his helpful suggestions.

\section{REFERENCES}

1. J. G. Hocking and G. S. Young, Topology, Addison-Wesley, Reading, Mass., 1961. MR 23 \#A2857. \#1007.

2. E. H. Spanier, Algebraic topology, McGraw-Hill, New York, 1966. MR 35

3. G. P. Weller, The intersection multiplicity of compact $n$-dimensional metric spaces, Proc. Amer. Math. Soc. 36 (1972), 293-294. MR 46 \#6314.

DEP ARTMENT OF MATHEMATICS, UNIVERSITY OF ILLINOIS AT CHICAGO CIRCLE, CHICAGO, ILLINOIS 60680 